\title{
3D FABRICATION
}

\section{Behold the fold}

Nature uses folding to create complex and intricate structures, with sizes ranging from protein molecules to plants. Scientists have been able to imitate some of these processes on small scales by exploiting, for example, DNA folding or surface-tension effects on lithographically patterned structures. Being able to form 3D shapes quickly and cheaply by simple printing techniques, however, is still difficult, and impossible for unsupported 'overhanging' features.

Now, a team led by Jennifer Lewis from the University of Illinois at UrbanaChampaign have taken inspiration from origami, the Japanese art of paper folding, to create a variety of 3D shapes from directly printed sheets (pictured, scale bars $=2 \mathrm{~mm}$; Adv. Mater. 22, 2251-2254; 2010). The 'ink' was a highly concentrated suspension of titanium hydride, stabilized by a triblock copolymer and a solvent system with graded

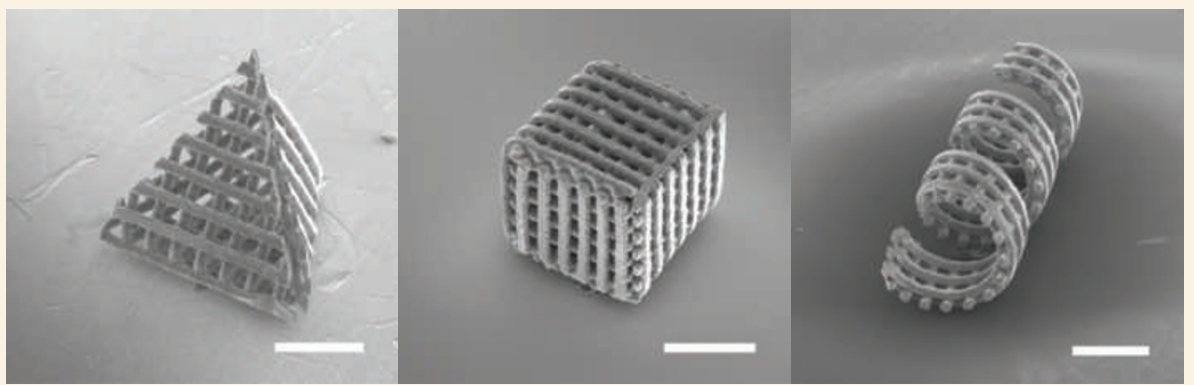

volatility. The printed, lattice-like sheets could be manipulated into complex 3D architectures that would otherwise not be possible to create through direct printing or lithography.

By controlling the volatility of the solvent system, Lewis and colleagues could control how long the sheets remained foldable - from 30 minutes to several hours. They made tetrahedral and cubic cages, concentric cylinders, helices and even an origami crane. The structures could be converted to either titanium or titanium dioxide by annealing at about $1,000^{\circ} \mathrm{C}$ in vacuum or air, respectively.

\section{NEIL WITHERS}

The original version of this story first appeared on the Research Highlights section of the Nature Chemistry website. 